\section{An Over-the-row Micronized-dust Applicator for Fruit Trees}

\author{
Charles L. Wilson', \\ Michael E. Wisniewski ${ }^{1}$, and
}

Bruce L. Upchurch ${ }^{2}$

Additional index words. pesticide, reduced drift, biological control

Summary. An apparatus was designed and built to deliver micronized dust particles to the foliage of mature orchard trees under a mobile canopy. The dust is propelled by compressed air (about $1120 \mathrm{kPa}$ ), which is pulsed through sand-blast guns using the Bernoulli effect. The canopy consists of steel pipes that support the cover and serve as a conduit for compressed air that flows to the guns. Quickcoupling fittings on the canopy pipes allow for easy attachment and removal of multiple guns at various, optional positions. The support structure for the canopy is attached to a mobile trailer, which transports it over mature orchard trees while the dust is being applied. The canopy reduces drift and enhances the coverage of dusts while they are being applied. This innovative apparatus can be used to apply pesticides, growth promoters and regulators, fertilizers, and biocontrol agents in powdered form. The distribution of corn starch on apple foliage is assessed using this invention.

$\mathrm{P}$ esticides were applied to fruit trees as sprays and dusts during the early years of commercial tree fruit production. With the advent of improved air-driven speed

USDA-ARS, Appalachian Fruit Research Station, 45 Wiltshire Road, Kearneysville, WV25430.

${ }^{1}$ Research plant patholoflist.

${ }^{2}$ Agricultural engineer.

We express our gratitude for the excellent technical assistance provided by Bill Anger, and Warren LaRue. The cost of publishing this paper was defrayed in part by the payment of page cbarges. Under postal reoulations, this paper therefore must be hereby marked advertisement solely to indicate this fact. sprayers and better spray formulations in the 1950s and 1960s, dusting decreased as a method for applying pesticides to trees (Childers, 1978). Recently, the application of pestcides Outdoor applications of dusts have been limited to sprays. Dust applications are made primarily indoors for controlling external parasites on livestock, poultry, and pets (Anon, 1990). Outdoor applications of dusts have been limited because they are susceptible to drift under windy conditions.

The application of dusts rather than sprays to fruit trees could be advantageous, however. If properly applied, dust applications might 1) reduce soil, air, and water contamination; 2) allow the application of chemicals that are difficult to solubilize; 3 ) reduce the amount of material necessary for efficacy; 4) improve the coverage of plant surfaces; 5) allow the use of more lightweight equipment, thus reducing soil compaction; and 6) elimi nate the need for water to make applications.

Our objective was to develop a means of applying dusts to trees with minimal drift. This paper describes the apparatus that we developed and presents preliminary test results showing dust distribution within the canopy when using this device. U.S. patent no. 5,288,621 has been issued covering this invention (Wilson and Wisniewski, 1993).

\section{Materials and methods}

A field micronized-dust applicator was constructed to cover mature semidwarf orchard trees with a canopy and apply micronized dust under the canopy to tree foliage (Figs. 14). The dust is delivered with sand-blast guns, which siphon the dust out of containers by the Bernoulli effect as compressed air is released through the gun aperture (Figs. 4-6).

The canopy. The canopy is semicircular in cross-section and is large enough to contain mature semidwarf apple trees (Fig. 1A). It is $4.25 \mathrm{~m}$ high, $4.25 \mathrm{~m}$ wide, and $4.25 \mathrm{~m}$ long. It is constructed of bent steel pipe that supports the canopy cover and provides a conduit for compressed air that is generated by a compressor on the trailer (Fig. $1 \mathrm{D}$ ). The arching vertical pipe members of the canopy are interconnected by a horizontal pipe that allows the entire supporting structure to be pressurized by one piece of pres- 
sure hose that runs from the compressor to the canopy-support pipe. The canopy is covered by ripstop polyethylene plastic lashed to the canopy pipes (Fig. 6).

The supported transport system. The canopy is supported by a steel structure that is attached to a trailer (Fig. 1 B ). It is suspended at the terminal end of its apex by chains that are attached to an I-beam. A cable and pulley system (Fig. 11) attached to the canopy support system allows the canopy to be extended and suspended beyond the trailer to cover trees and retracted over the trailer for transport.

The pneumatic generation and conduction system. An air compressor (Fig. 1D ) on the trailer capable of generating $1400 \mathrm{kPa}$ pressure is attached by a single hose to the canopy pipes (Fig. 1H). Quick-couplers (Fig. $1 \mathrm{~K})$ on the inside of the canopy support pipes allow the attachment of a variable number (1-1 O) of sand-blast guns (Fig. 6) to the pneumatic pipes of the canopy (Fig. 5).

Sand-blast guns and duet con- tainers. Sand-blast guns are mounted to the canopy pipe with C-clamps and attached by hoses to the pneumatic pipes in the canopy (Figs. 5 and 6). The dust container (Figs. 1L and 7) is constructed of PVC sewer pipe, with pipe caps on the bottom and top. A hose is attached from the bottom of the dust container to the siphoning inlet of the dust gun. A solenoid valve (Fig. 5D) is placed on the pneumatic conduit from the canopy pipe to the dust gun. The opening and closing of this valve pulses air through the sandblast gun.

Electrical system. The duster electronic circuits control dust application quantity and rate. In addition, pulsing the duster guns improved dust flow from the storage containers and reduced nozzle clogging. A diagram of the control circuit for the solenoid valves is shown in Fig. 7. Pulsing of the duster guns is initiated by the manual start switch. This switch is toggled onoff when a tree is enclosed in the canopy. The manual start switch initiates the single-shot output of the cycle limit relay (SSAC $\mathrm{p} / \mathrm{n}$ TDSL), selectable from 0.1 to $102.3 \mathrm{sec}$ in 0.1 sec increments. This time-interval relay limits the length of the cycle time for the duster guns. Normally, this time is set for the time the tree remains

Fig. 1. The over-the-row micronized-dust applicator. (A) Canopy consisting of interconnected bent pipe covered with tearproof plastic. (B) Support structure from which canopy is suspended by short chains. Canopy can be retracted over the trailer by a cable and pulley (I) for transport. (C) Trailer with compressor $(D)$, battery $(E)$, the electronic circuit box $(G)$, and wires from the battery to the electronic circuit box (F). (H) A pressure hose running from the compressor to the pipes supporting the canopy. All pipes comprising the canopy support system are interconnected, which allows the entire canopy support system to be pressurized. (J) Wires running from the circuit control box to the solenoid valves. (K) Quick-couplers on the pressurized conduit pipe, which serves as support for the canopy cover $(A)$. These allow the addition of guns or the repositioning. of existing guns. (L) Canisters that hold the powdered material. They are constructed of PVC sewerpipe and caps. (M) Tips of sandblast gunsv from which the dust is expelled can be seen protruding inside the canopy.

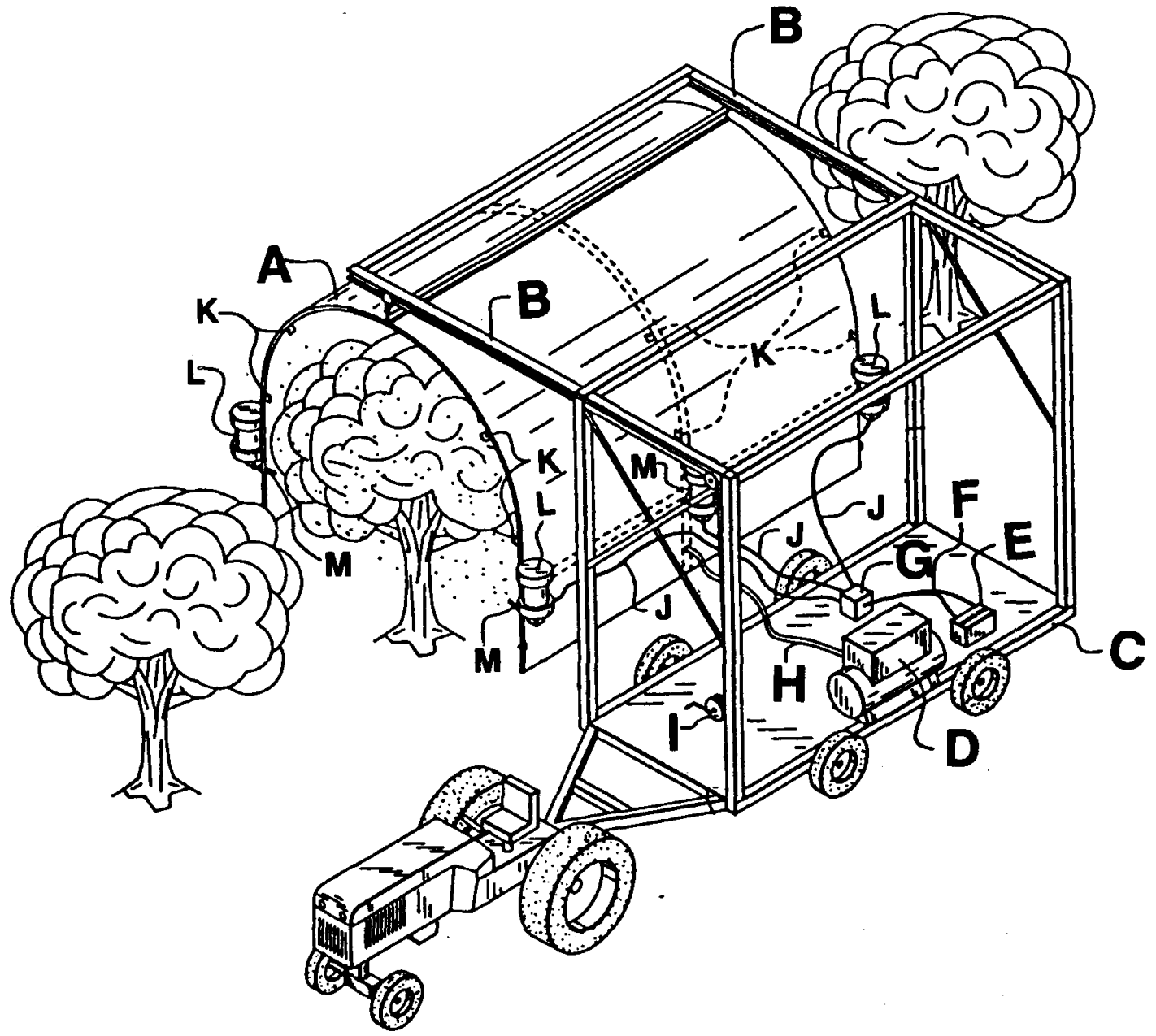


Fig. 2. Overall endview of canopy, Support Structure, and transport trailer.

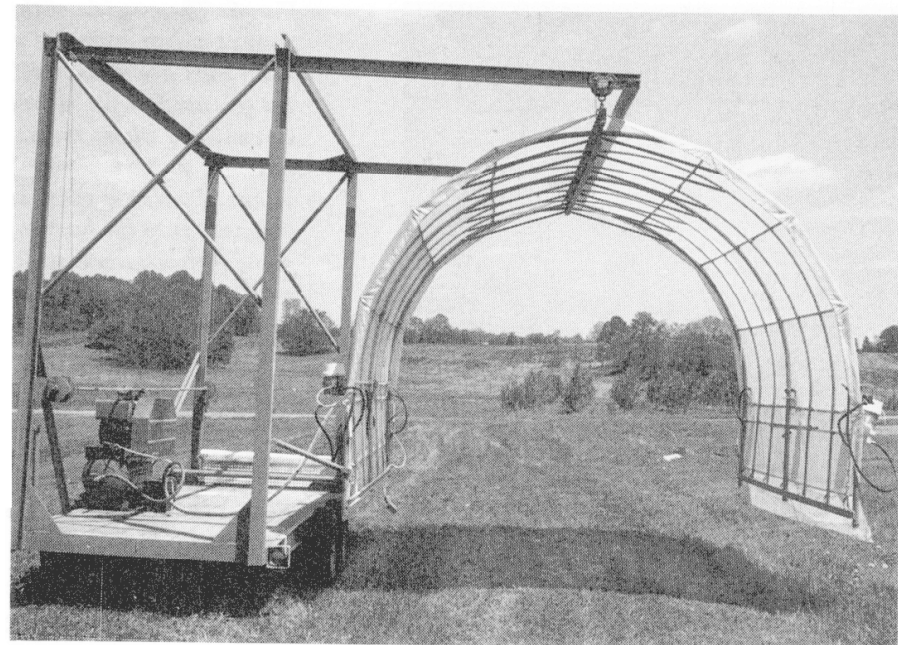

Fig. 3. Overall side-view of compressor, transport trailer, support structure, and canopy.

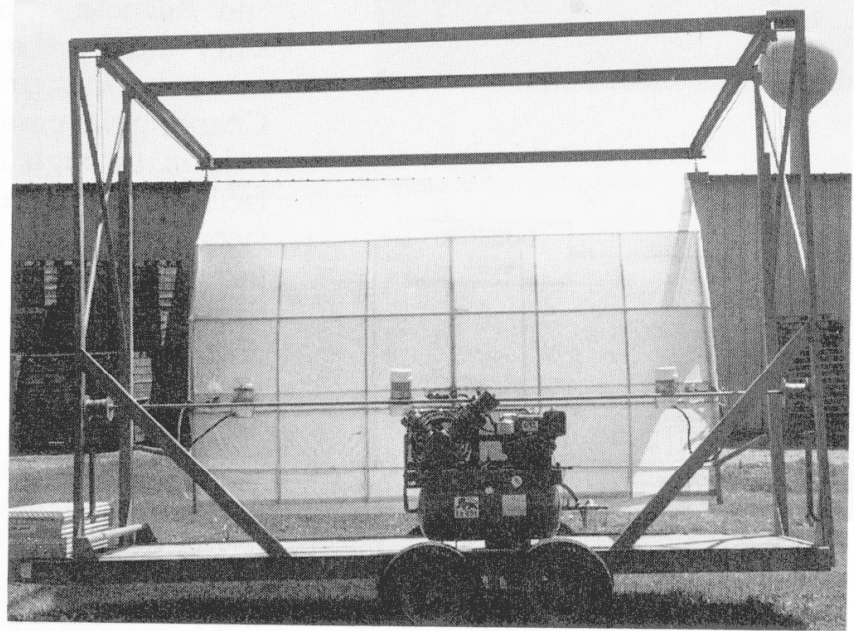

Fig. 4. Guns protruding under the canopy and extra quick-couplers, which allow repositioning or adding more guns.

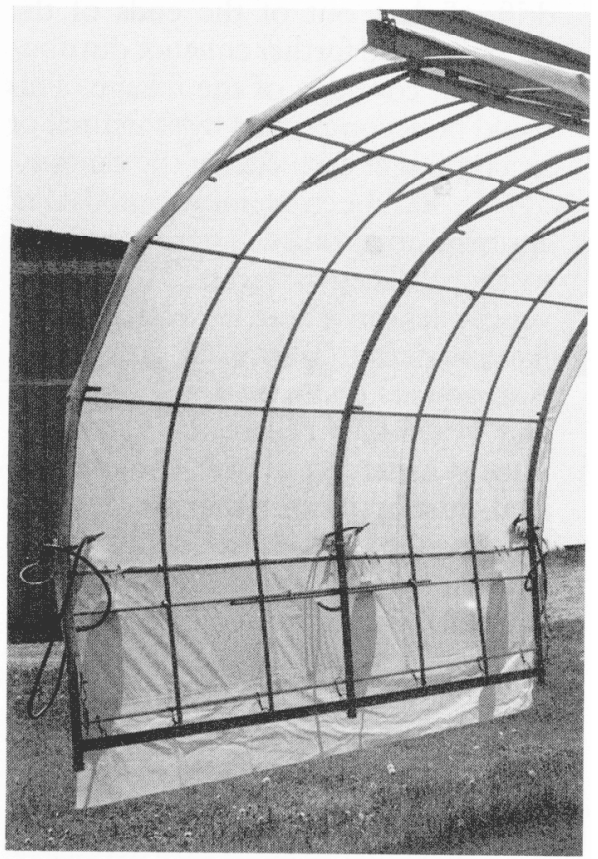

within the canopy. A LM555 timer circuit in conjunction with five pulse interval relays (SSAC $\mathrm{p} / \mathrm{n}$ TDSL) determines the pulse rate of the duster

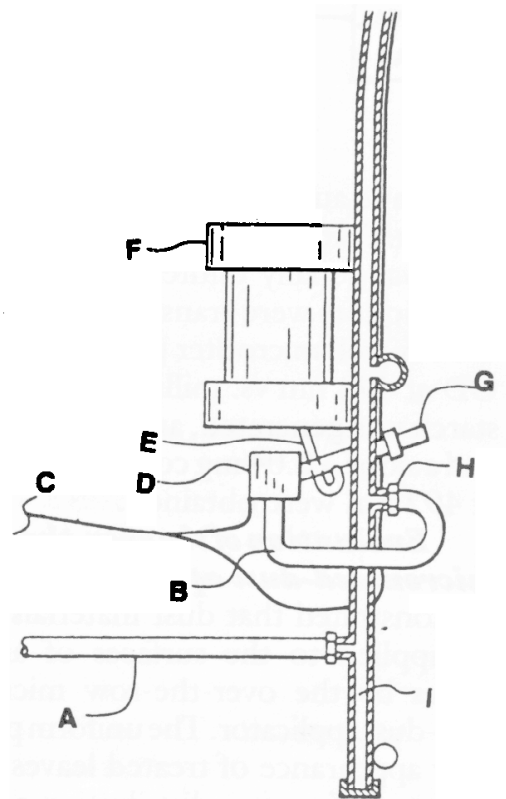

guns. The gun pulsing rate is adjusted with a potentiometer in the timing circuit that changes the reset timing rate of all five of the pulse rate relays. In addition, the pulse rate is adjusted for each pair of guns by the selectable settings on each pulse interval relay. These adjustments are set for uniform dust coverage.

Power for the electronic control circuits is provided by a battery in the pulling tractor. A quick-disconnecttype connector at the rear of the tractor wired to the tractor battery and a mating connector at the front of the canopy wired to a control box simplify power availability. A rainproof control box mounted on the trailer (Fig. 1G) houses all electronic components. Two conductor-control cables connect each duster-gun solenoid to the pulse-rate relay in the control box (Fig. 1J).

Procedures for evaluating corn starch dust distribution. Whole leaves were collected 1 and $3 \mathrm{~m}$ above the ground from trees where corn starch (Dial Corp., Phoenix, Ariz.) had been applied with the micronizeddust applicator. Samples (10 leaves at each collection site) were collected at random from each of four quadrants at each sampling level. These leaves were taken, $0.2 \mathrm{~m}$ from the edge of the canopy at each sampling location. Three trees were sampled.

Ten leaves from each collection site were placed in individual zip-lock plastic bags. Ten milliliters of water was added to each bag, and each leaf was washed vigorously by shaking the bag. The washings were transferred to glass test tubes and autoclave for $5 \mathrm{~min}$ to dissolve the corn starch. A 100-ml aliquot of the washing was transferred to wells of a 96-well microtiter plate, and $15 \mathrm{ml}$ of a $5 \%$ IKI solution $(\mathrm{w} / \mathrm{v})$ was added to each well. The plates

Fig. 5. Dust-containing canister, solenoid valve, and dust gun attached to a crosssection of pipe in the canopy support system. (A) Pressure hose runningfrom the compressor to a pipe (I) in the canopy support system. (B) Pressure hose running. from the canopy support pipe to the solenoid valve (D). (E) Hose running. from the dust-containing canister to the barrel of the dust gun (G). When the solenoid valve opens, pressurized air is released into the gun barrel, which siphons dust from the dust canister by tbe Bernoulli effect. (C) Wires running to the electronic circuit box which controls the pulsing of the solenoid valve. 

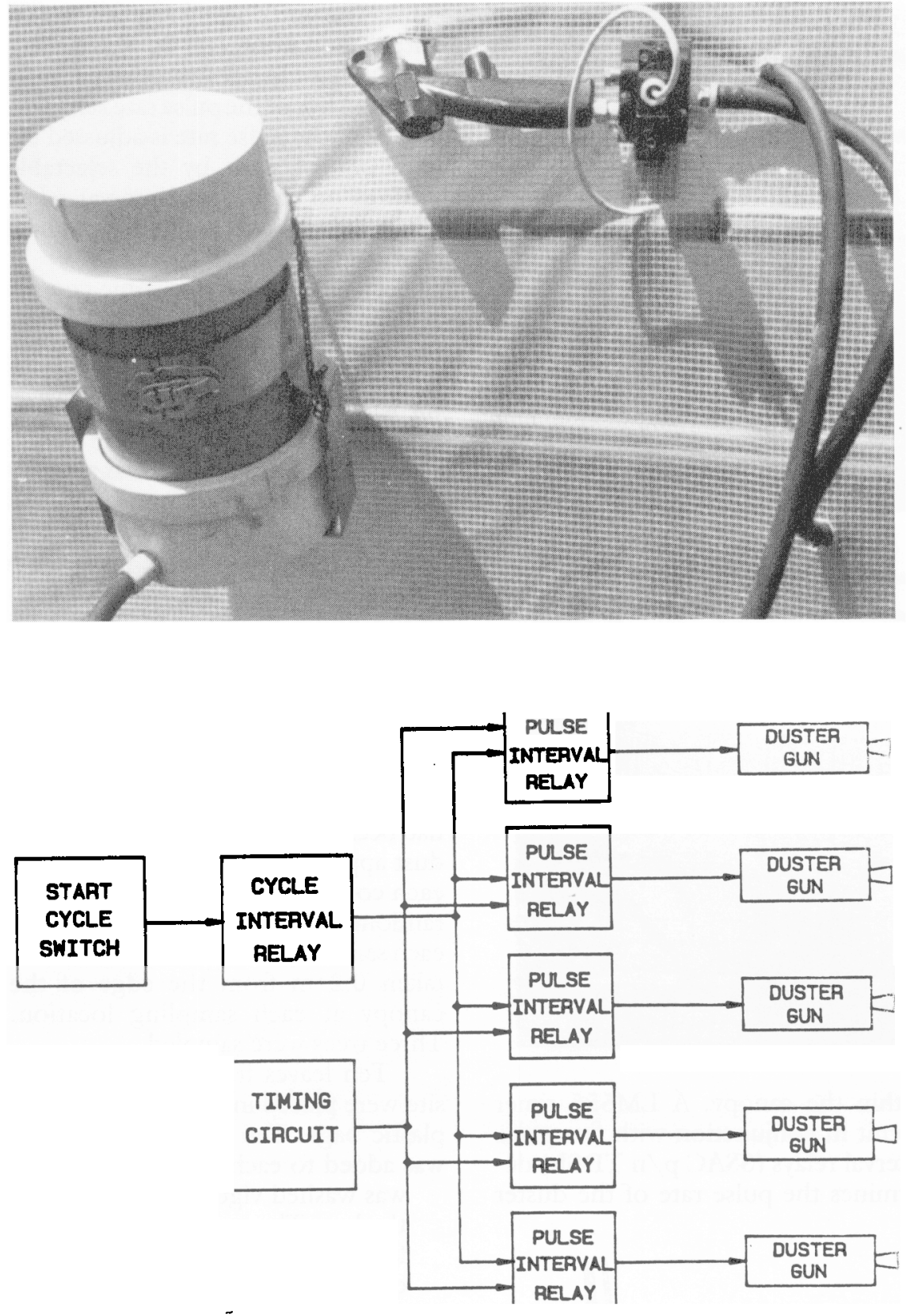

Fig. 7. General circuitry in the electronic circuit box to contvol the pulsing of the solenoid valves connected to the dust guns.

were shaken for 1 rein, and the optical density (OD) of each well was read on an plate reader (EAR 400T; STL, San Francisco) at $492 \mathrm{~nm}$. Leaf surface area was measured using an area meter (LI-COR, Lincoln, Neb.) and doubled to include both sides of the leaf. The corn starch deposition data are reported as $\mathrm{mg}$ corn starch $/ \mathrm{cm}^{2}$ leaf surface (Table 1).

A standard curve for $\mathrm{mg}$ corn starch/OD at $492 \mathrm{~nm}$ was prepared by making a $0.3 \%$ stock solution in water
Fig. 6. The outside of the canopy where dust canister, solenoid valve, and sandblast gun are attached. The wire from the solenoid valve runs the electronic circuit box where the pulsing of the solenoid valve is controlled. When the solenoid valve is open, compressed air is released into the barrel of the sand-blast gun, which siphons dust from the canister (using the Bernoulli effect) and expels it into the canopy.

dust on leaf surfaces (Fig. 8). Also, leaves treated with the micronizeddust applicator had a uniform blue color over the leaf surface when dipped in a $2 \%$ IKI solution (data not shown).

As dust particles are propelled out of the dust guns. they become electrostatically charged by friction (Brazee and Buchele, 1959). Mutual repellency of charged starch particles could account for their even distribution. Charging the particles by pulsed propulsion through the guns also may facilitate their adhesion to the leaf surfaces. The pulsing action also creates a turbulence under the duster canopy that facilitates the distribution of the dust.

\section{Conclusion}

In our preliminary tests using powdered corn starch, we found corn starch distributed evenly throughout the tree canopy (Table 1). Uniform deposits were evident on individual leaves throughout the tree (Fig. 8). The static electrification of the dust particles probably accounts for their even distribution and adherence to leaf surfaces (Kunkel, 1950). It is assumed that restricting the drift of dust out of the ends of the canopy would further enhance distribution and coverage of the foliage. This could be accomplished by structural or air curtains at each end of the canopy.

It has been demonstrated that applying antagonistic microorganisms to the surfaces of harvested fruit and vegetables can reduce losses from postharvest decay (Wilson et al., 1991). Antagonists could be applied to fruit and vegetables before harvest to reduce postharvest decay. The micronized-dust applicator that we describe here could be used to deliver Powdered preparations of antagonists to fruit surfaces before harvest. Dried formulations of microorganisms may be more desirable than liquid formulations because the organism would remain dormant until it reaches the target site.

An ability to apply powdered pes- 
Table 1. Distribution of the deposition of corn starch ( $m \mathrm{~g}^{\mathrm{c}} \mathrm{cm}^{-2}$ leaf surface), within the canopy of three semidwarf 'Rome Beauty' apple trees, applied by the over-the-row micronized-dust applicator traveling at about $5 \mathrm{~km} \cdot \mathrm{h}^{-1}$. Quantities represent an average of 10 leaves sampled at 1 or $3 \mathrm{~m}$ from the ground in each of four quadrants. Samples were taken $0.2 \mathrm{~m}$ from the edge of the canopy.

\begin{tabular}{lcccc}
\hline \multicolumn{2}{c}{ Sample location } & & \multicolumn{3}{c}{ Tree } \\
\cline { 4 - 5 } Quadrant & Ht & & 1 & 3 \\
\hline I & 1 & $25.1 \pm 0.8^{\mathrm{z}}$ & $24.3 \pm 0.8$ & $23.0 \pm 0.4$ \\
& 3 & $21.0 \pm 0.6$ & $19.6 \pm 0.5$ & $23.5 \pm 0.4$ \\
2 & 1 & $31.4 \pm 0.4$ & $30.0 \pm 0.4$ & $20.2 \pm 0.3$ \\
& 3 & $21.4 \pm 0.4$ & $19.2 \pm 0.4$ & $31.8 \pm 0.7$ \\
3 & 1 & $19.5 \pm 0.6$ & $20.2 \pm 1.5$ & $15.1 \pm 0.1$ \\
& 3 & $24.9 \pm 0.9$ & $21.9 \pm 0.8$ & $24.3 \pm 0.5$ \\
4 & 1 & $36.0 \pm 1.9$ & $26.7 \pm 0.6$ & $19.3 \pm 0.3$ \\
& 3 & $35.4 \pm 1.4$ & $23.3 \pm 0.7$ & $24.6 \pm 0.4$ \\
\multirow{2}{*}{ Whole tree } & & $26.6 \pm 6.6$ & $23.1 \pm 3.8$ & $22.6 \pm 4.9$ \\
\hline
\end{tabular}

${ }^{2}$ Mean $\pm S D, n=10$.

${ }^{y}$ Grand mean $\pm S D$ derived from the average of all eight sampling locations, $\mathrm{n}=80$.

Fig. 8. (A) Typical apple leaf from tree dusted with corn starch with micronized-dust applicator showing uniform distribution of dust on leaf surface. (B) Two vows of semidwarf 'Rome Beauty' apple trees. The row On the left was dusted with the over-the-vow micronized-dust applicator and the row on the right was untreated. Distribution of the corn starch within three of the treated trees is shown in Table 1. (C) Canopy covering semidwarf 'Rome Beauty' apple tree. Note containment of the dust being discharged from guns. (D) Canopy covering semidwarf 'Rome Beauty' apple tree. Trajectory of dust from the gun on the left side of the canopy is

\section{Literature Cited}

Anonymous. 1990. Farm chemicals handbook. Dry applications equipment. Meister Publ. Co., Willoughby, Ohio.p.311-313.

Brazee, R.D. and W.F. Buchele 1959. Electrostatic precipitation of pesticidal dusts, an outline of research and literature. USDA42-29.

Childers, N.F. 1978. Modern fruit sciences. Horticulture Publ., New Brunswick, N.J.

Kunke, W.B. 1950. The static electrification of dust particles on dispersion into a cloud. J. Applied Physics 21:820-832.

Wilson, C.L. and M.E; Wisniewski 1993. Apparatus and method for applying material to agricultural commodities. U.S. patent no. $5,228,621$.

Wilson, C. L., Wisniewski, M. E., Bales, C. L., McLaughlin, R., Chalutz, E., and S. Droby. 1991. Biological control of post-harvest diseases of fruits and vegetables: alternatives to synthetic fungicides. Crop Protection 10:172-177.

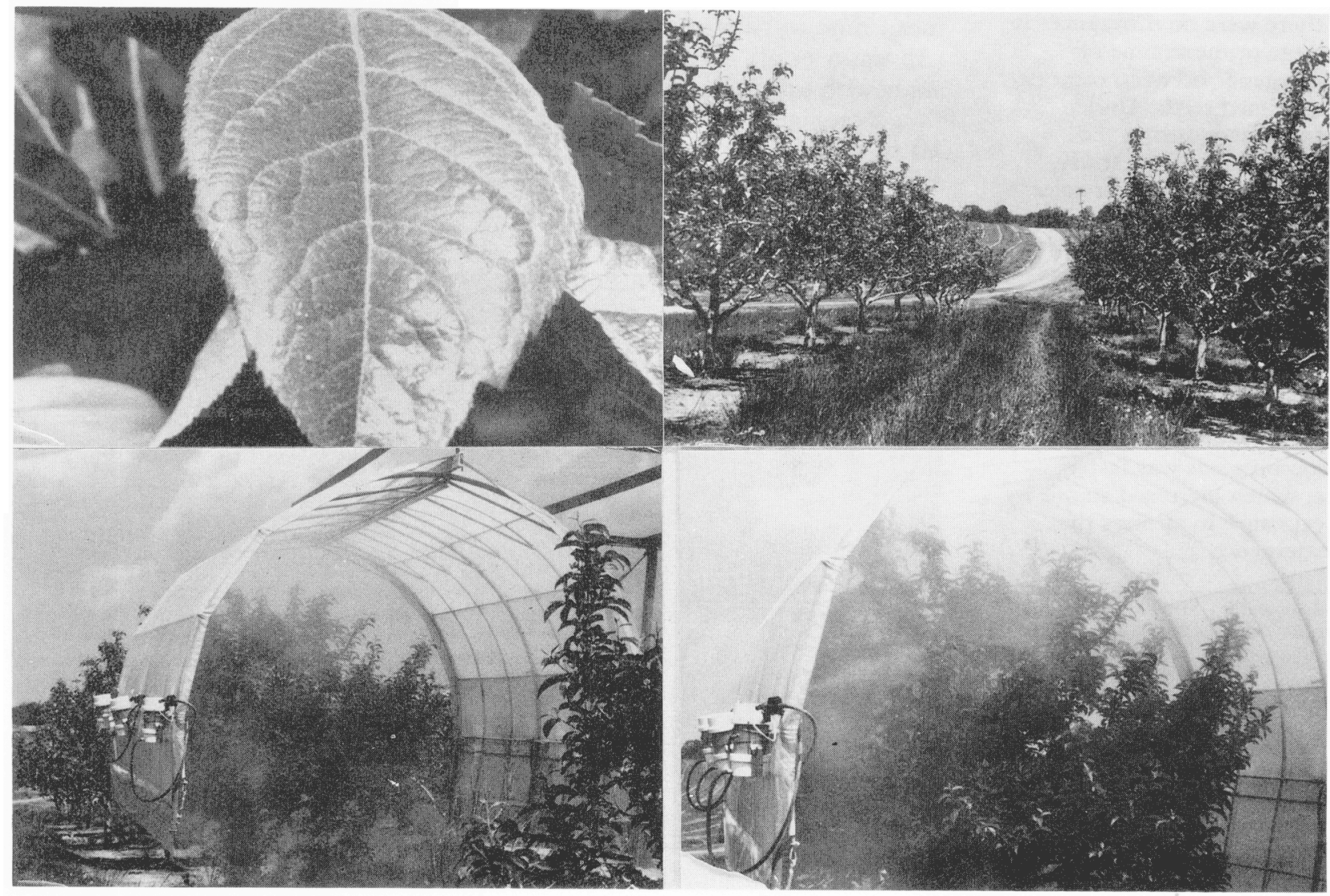

